\title{
Conductance through a one-dimensional correlated system: Relation to persistent currents and the role of the contacts
}

\author{
Rafael A. Molina, ${ }^{1,2}$ Dietmar Weinmann, ${ }^{1}$ Rodolfo A. Jalabert, ${ }^{1}$ Gert-Ludwig Ingold, ${ }^{3}$ and Jean-Louis Pichard ${ }^{2}$ \\ ${ }^{1}$ Institut de Physique et Chimie des Matériaux de Strasbourg, \\ UMR 7504 (CNRS-ULP), 23 rue du Loess, BP 43, 67034 Strasbourg Cedex 2, France \\ ${ }^{2}$ CEA/DSM, Service de Physique de l'Etat Condensé, Centre d'Etudes de Saclay, 91191 Gif-sur-Yvette, France \\ ${ }^{3}$ Institut für Physik, Universität Augsburg, Universitätsstraße 1, 86135 Augsburg, Germany \\ (Received 9 August 2002; revised manuscript received 12 March 2003; published 10 June 2003)
}

\begin{abstract}
Based on a recent proposal [O.P. Sushkov, Phys. Rev. B 64, 155319 (2001)], we relate the quantum conductance through a sample in which electrons are strongly correlated to the persistent current of a large ring, composed of the sample and a noninteracting lead. A scaling law in the lead length allows to extrapolate to a well-defined value of the conductance, depending only on intrinsic properties of the sample and the nature of the contacts between the sample and the lead. For strongly disordered samples, the conductance is found to be enhanced by the interaction.
\end{abstract}

DOI: 10.1103/PhysRevB.67.235306

PACS number(s): 73.23.-b, 05.60.Gg, 71.10.-w, 73.63.Nm

Viewing quantum transport as a scattering problem ${ }^{1,2}$ generated a new understanding of the electronic conductance. This approach is able to explain a wealth of experimental results $^{3}$ in mesoscopic systems when electron-electron $(e-e)$ correlations are not important. To include these correlations is nontrivial and remains one of the major challenges in the field (see, e.g., Ref. 4). While none of the proposals to calculate the conductance for a correlated system is well suited for numerical calculations ${ }^{5,6}$ or free of certain assumptions, ${ }^{7}$ such an issue becomes crucial in present day's research exploring electronic transport through nanosystems (carbon nanotubes, ${ }^{8}$ molecules, ${ }^{9}$ and point contacts $^{10}$ ), where the Coulomb repulsion leads to important correlations.

Reservoirs and leads are key elements in the scattering approach, and possess very clear physical meanings since the measurement is made with electrodes that behave as electron reservoirs. In a good electrode, the electron density $n_{e}$ is large, the ratio $r_{s}$ between the Coulomb and the Fermi energy is small, and hence the $e-e$ interaction is negligible. In contrast, $n_{e}$ in a nanosample can be very small, yielding a large ratio $r_{s}$ and important $e$-e correlations.

The dimensionless conductance $g$ does not only depend on the intrinsic properties of the sample, but also on the way it is connected to the electrodes. The quality of the contacts is particularly important for correlated electrons. For a clean Luttinger liquid attached to noninteracting leads through reflectionless contacts, it has been found ${ }^{11}$ that the interactions do not influence $g$. In the other extreme, if the contacts are tunnel barriers, the interactions lead to the Coulomb blockade, ${ }^{12}$ thereby dominating $g$. In carbon nanotubes, various transport regimes are observed depending on the nature of the contacts. ${ }^{8}$

As shown by Kohn ${ }^{13}$ and Thouless, ${ }^{14} \mathrm{~g}$ is also related to the sensitivity of the sample's eigenstates to a change of the boundary conditions. This sensitivity can be tested by closing a system to a ring and measuring the persistent current as the response to an enclosed magnetic flux $\phi$. At zero temperature, the persistent current is given by $J=-\partial E / \partial \phi$, where $E$ is the ground-state energy of the many-body system.
Interactions play an important role for $J$, and it is generally accepted that they account for the large difference between experiments and one-particle calculations. ${ }^{15}$ There have been various attempts ${ }^{7,16}$ to link $J$ and $g$ for an interacting ring. However, the ring built from the sample itself does not contain any reservoirs in which energy relaxation can take place. Negative zero-frequency conductivities occur, ${ }^{17}$ unlike in the dissipative case in which we are interested here.

As pointed out in Refs. 5 and 10, at zero temperature, not only for the noninteracting case, but also for correlated samples, $g$ is given by $\left|t\left(E_{F}\right)\right|^{2}$, the probability for an electron at the Fermi energy $E_{F}$ to be elastically transmitted through the sample. Moreover, if one replaces the massive electrodes (with negligible $e$-e correlations) used in a real measurement by very long noninteracting one-dimensional leads, one can expect that they have a similar effect. ${ }^{6}$ Sushkov recently proposed ${ }^{10}$ that $\left|t\left(E_{F}\right)\right|^{2}$ can be extracted from the persistent current of a much larger ring, composed of the sample itself, together with a long lead closing the system. This has the considerable advantage that a ground-state property $(J)$ suffices to determine $g$. However, one needs the $J$ of the combined system (sample plus lead), and not the one of the system alone as in previous works. ${ }^{7,13,14,16}$

In the following, we adapt the approach of Ref. 10 to calculate $g$ for one-dimensional interacting electrons using the density-matrix renormalization-group (DMRG) algorithm. ${ }^{18,19}$ We check that a scaling law allows to extrapolate to an infinite lead, yielding $g$ as a property of the sample and the way it is connected to the lead. The nature of the contacts turns out to play a major role. Then, we extend our analysis to disordered samples, where we find that, similarly to the case of persistent currents, ${ }^{20}$ repulsive interactions may increase $g$ for strong disorder.

We first present an alternative derivation of Sushkov's result, ${ }^{10}$ pointing out the main assumptions, for the noninteracting case. As depicted in the upper inset of Fig. 1, we consider a sample ( $S$, hashed region) closed to a ring by a noninteracting and disorder-free lead $(L)$, and threaded by a flux $\phi$. The total length $L=L_{\mathrm{S}}+L_{\mathrm{L}}$ consists of the sample 


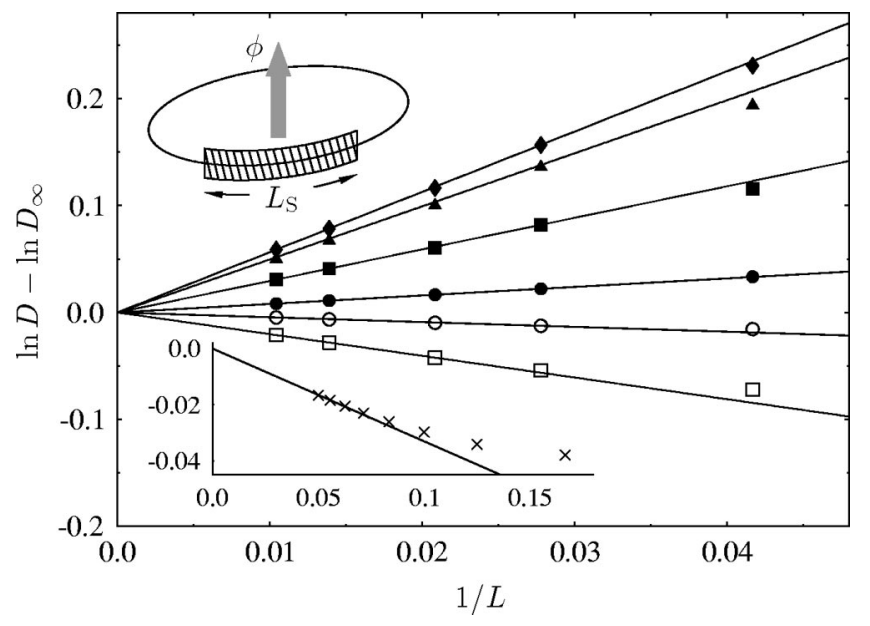

FIG. 1. Scaling of $\ln D$ with the total length $L$ of the system, showing a linear increase of $\ln D$ with $1 / L$ for even $L_{\mathrm{S}}=12[U$ $=1(\boldsymbol{\bigcirc}), U=2(\boldsymbol{\square}), U=3(\boldsymbol{\Delta}), U=4(\diamond)]$, and a decrease for odd $L_{\mathrm{S}}=13[U=1(\bigcirc)$ and $U=2(\square)]$. Lower inset: scaling for unpolarized electrons within the Hubbard model $\left(L_{S}=2, U=1\right)$. Upper inset: sketch of a ring consisting of the sample (hashed region) and a noninteracting lead threaded by a flux $\phi$.

length $L_{\mathrm{S}}$ and the lead length $L_{\mathrm{L}}$. The one-particle eigenstates of the total system satisfy

$$
\operatorname{det}\left(I-M_{\mathrm{S}} M_{\mathrm{L}}\right)=0,
$$

with the transfer matrices of the sample and the lead,

$$
\begin{gathered}
M_{\mathrm{S}}=\frac{1}{\sin \varphi}\left(\begin{array}{cc}
e^{i \alpha} / \sin \theta & -i \cot \theta+\cos \varphi \\
i \cot \theta+\cos \varphi & e^{-i \alpha} / \sin \theta
\end{array}\right), \\
M_{\mathrm{L}}=e^{i \Phi}\left(\begin{array}{cc}
e^{i k L_{\mathrm{L}}} & 0 \\
0 & e^{-i k L_{\mathrm{L}}}
\end{array}\right),
\end{gathered}
$$

respectively. Here, $\Phi=2 \pi \phi / \phi_{0}$ where $\phi_{0}$ is the flux quantum. The scattering is characterized by the angle $\theta$, the phase shift $\alpha$, and the angle $\varphi$ (equal to $\pi / 2$ if right-left symmetry is respected). These angles are functions of $k$, the wave vector in the lead. The transmission amplitude is given by $t$ $=e^{i \alpha} \sin \theta \sin \varphi$. With Eq. (2), the quantizing condition (1) can then be written as

$$
\cos \Phi=\frac{1}{|t|} \cos (k L+\delta \alpha),
$$

with the relative phase shift $\delta \alpha=\alpha-k L_{\mathrm{S}}$. The persistent current carried by a one-particle state (with energy $\epsilon$ ) is $j(\phi)=-(\partial \epsilon / \partial k)(\partial \phi / \partial k)^{-1}$. We work at $\Phi=\pi / 2$ and establish two crucial assumptions: (i) $|\partial(\delta \alpha) / \partial k| \ll L$, (ii) $\partial \epsilon / \partial k \simeq \hbar^{2} k / m$. The first one states that the Wigner time associated with the scattering region is negligible compared with the time spent in the leads. Notice that we work with a relative Wigner time $\tau_{W}=\left(m / \hbar^{2} k\right) \partial(\delta \alpha) / \partial k$, that is, the difference between the delay time of the scattering region and that of a potential-free region having the same length. The second assumption implies that the dispersion relation is essentially unaffected by the scattering potential.
The persistent current of $N$ noninteracting spinless fermions (for simplicity we take $N$ even) is given by ${ }^{21}$

$$
J(\Phi=\pi / 2)=\frac{e \hbar}{m L} k_{F}\left|t\left(k_{F}\right)\right| .
$$

Therewith, the conductance may be related to the ratio of $J(\Phi=\pi / 2)$ to the persistent current $J^{0}$ of a clean ring of length $L .{ }^{10}$ This relation becomes exact in the limit of infinite lead length when the above assumptions hold, and one gets

$$
g=\lim _{L_{\mathrm{L}} \rightarrow \infty}\left(\frac{J(\pi / 2)}{J^{0}(\pi / 2)}\right)^{2} .
$$

With interaction, ${ }^{22}$ assumptions (i) and (ii) always hold in the large $L$ limit. Moreover, the use of Eq. (5) implies that the one-particle states of the correlated system can still be indexed by the lead wave vectors $k$. That is, adding an infinite noninteracting lead to a finite non-Fermi-liquid sample restores the Fermi-liquid behavior. This assumption, which has been used in the perturbative calculation of transport through Hubbard chains connected to reservoirs, ${ }^{23}$ requires that the interactions are completely switched off in the onedimensional lead. Otherwise the Luttinger liquid behavior ${ }^{24}$ sets in, and one cannot obtain a result that is independent of the length of the auxiliary lead. In this, our approach differs from Sushkov's, where the interactions in the lead are kept (within the Hartree-Fock approximation).

Equation (5) allows to calculate $g$ from the ground-state energies. We do this for spinless fermions (polarized electrons) in a ring described by the Hamiltonian

$$
H=-\sum_{i=1}^{L}\left(c_{i}^{\dagger} c_{i-1}+c_{i-1}^{\dagger} c_{i}\right)+\sum_{i=2}^{L_{S}} U\left[n_{i}-\frac{1}{2}\right]\left[n_{i-1}-\frac{1}{2}\right],
$$

where $c_{i}\left(c_{i}^{\dagger}\right)$ is the annihilation (creation) operator at site $i$, $n_{i}=c_{i}^{\dagger} c_{i}$ is the number operator, and the flux-dependent boundary condition enters through $c_{0}=\exp (i \Phi) c_{L}$. The interaction is restricted to nearest neighbors and effective in the sample, but vanishing in the lead. It is equilibrated by a compensating potential that prevents the particles from emptying the interacting region. The form of the Hamiltonian allows to have particle-hole symmetry at half filling. We work with a number of fermions $N=L / 2$, such that the mean density is always $1 / 2$ independently of $L_{\mathrm{S}}$ and $L_{\mathrm{L}}$.

Using the DMRG algorithm as described in Ref. 19, we calculate the ground-state energies $E(\Phi)$ at $\Phi=0$ and $\Phi$ $=\pi$, to obtain the stiffness $D=(L / 2)|E(0)-E(\pi)|$ (which is a measure for $J$ and simpler to calculate).

An obvious requirement for Eq. (5) to be useful is that the limit $L_{\mathrm{L}} \rightarrow \infty$ of the computed quantities is well defined. Therefore, the first numerical step is to compute $D$ for increasing $L_{\mathrm{L}}$ with given $L_{\mathrm{S}}$ and $U$ (Fig. 1). We find a very clear asymptotic behavior, described by the scaling law

$$
D\left(U, L_{\mathrm{S}}, L_{\mathrm{L}}\right)=D_{\infty}\left(U, L_{\mathrm{S}}\right) \exp \left(\frac{C\left(U, L_{\mathrm{S}}\right)}{L}\right),
$$




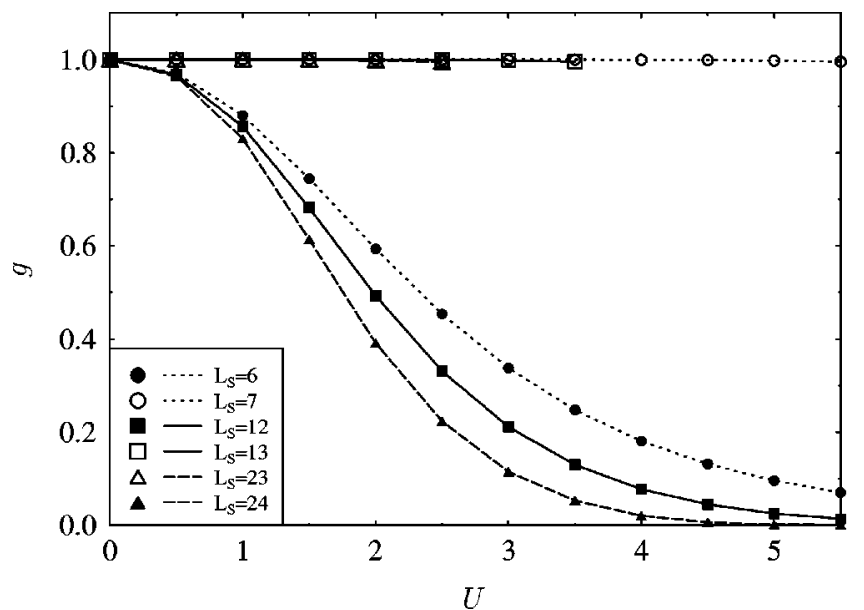

FIG. 2. Conductance $g$ as a function of the interaction strength $U$ for different values of the sample length $L_{\mathrm{S}}$.

where the intrinsic value $D_{\infty}$ is independent of the length of the auxiliary lead. The sign of $C$ depends on the parity of the number of sites $L_{\mathrm{S}}$ in the sample: $C>0$ for even $L_{\mathrm{S}}$ (filled symbols) and $C<0$ for odd $L_{\mathrm{S}}$ (open symbols). ${ }^{26}$

From the flux dependence of $J(\Phi)$ for a long clean chain containing a weak link, ${ }^{25}$ one gets the relation between $J(\pi / 2)$ and $D$. The asymptotic value $D_{\infty}$ is then used to determine conductance (5) as $g=\sin ^{2}\left[(\pi / 2)\left(D_{\infty} / D_{\infty}^{0}\right)\right]$, where $D_{\infty}^{0}$ corresponds to the clean noninteracting ring.

The method also works for nonpolarized electrons (the Hubbard model with on-site interaction), and the size scaling again allows to obtain intrinsic values (lower inset in Fig. 1). In the sequel we concentrate on the spinless case [Hamiltonian (6)], which contains the main features we are interested in, and allows to reach larger samples.

Having verified the consistency of our approach, we now study the systems of interest. In Fig. 2, we present the conductance, as a function of $U$, for various sample lengths $L_{\mathrm{S}}$. One observes a very clear even-odd asymmetry according to the parity of $L_{\mathrm{S}}$. Samples with odd $L_{\mathrm{S}}$ exhibit almost perfect transmission up to the largest values of $U$ for which the numerically reachable $L_{\mathrm{L}}$ allows for a reliable scaling to the limit $L_{\mathrm{L}} \rightarrow \infty$, while an even $L_{\mathrm{S}}$ results in a decrease of $g(U)$ already at weak interaction. For odd $L_{\mathrm{S}}$, particle-hole symmetry leads to degenerate sample configurations with $\left(L_{S} \pm 1\right) / 2$ particles in the interacting region. This is similar to a Coulomb blockade resonance. The traveling particle can thus become trapped for a long time $\left(\tau_{W}>0\right)$, consistent with negative $C .^{26}$ Since the two configurations are coupled by processes which transfer particles through the interacting sample, one obtains perfect transmission. A similar effect was found in the perturbative treatment of clean Hubbard chains. $^{23}$ The recovery of this limiting case is another consistency check of our method.

On the other hand, an even number of sites implies that the transport of one particle through the sample takes place via a virtual state with an energy of the order of $U$ above the ground state. Thus, no resonance can be expected and the transmission, which is suppressed already by moderate $U$, is

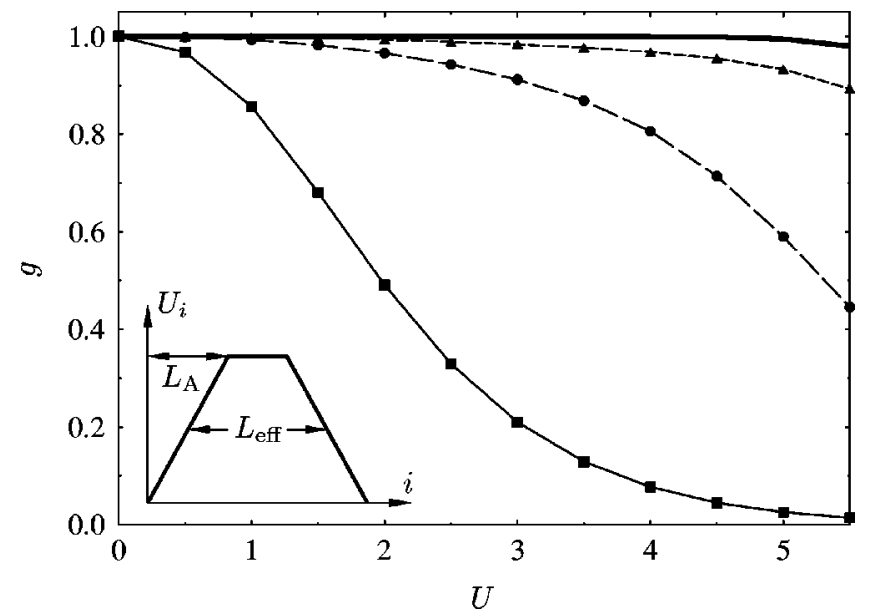

FIG. 3. Conductance $g$ as a function of the interaction strength $U$, for a fixed $L_{\text {eff }}=12$, and increasing smoothing of the contacts, defined by the length $L_{\mathrm{A}}$ (see inset). All data correspond to even $L_{\mathrm{S}}$ with $L_{\mathrm{A}}=0(\boldsymbol{\square}), L_{\mathrm{A}}=4(\boldsymbol{O})$, and $L_{\mathrm{A}}=10(\boldsymbol{\Delta})$. Using the same smoothing length $\left(L_{\mathrm{A}}=10\right)$ but improving in the shape [a tanh function (thick solid line) instead of a linear increase $(\boldsymbol{\Delta})]$ helps $g$ to approach the perfect value.

a fast process with $\tau_{W}<0$, consistent with $C>0$. In addition, increasing $L_{\mathrm{S}}$ reduces $g$ linearly for small $U$, and exponentially for $U>2$, consistent with the Mott-insulating behavior.

The even-odd asymmetry and the perfect transmission for the odd case point to the importance of the contacts. In order to investigate their role, we introduce a position-dependent interaction strength $U_{i}$ which increases linearly from 0 to its maximum value $U$, inside the "contacts" of length $L_{\mathrm{A}}$ (see inset of Fig. 3). As shown in Fig. 3, in the case of even $L_{\mathrm{S}}$ these smooth contacts increase $g$, at constant effective length $L_{\text {eff }}=L_{\mathrm{S}}-L_{\mathrm{A}}$ of the sample, and the conductance approaches the ideal situation of perfect transmission expected for reflectionless contacts when we improve the smoothing. ${ }^{11}$ The perfect transmission at odd $L_{\mathrm{S}}$ persists when the form of the contacts preserves the right-left symmetry, but it is destroyed by asymmetric contacts. The strong influence of the contacts is crucial when describing experiments since it seems impossible to connect a nanosample via reflectionless contacts. It also shows the limitation of other approaches relating the conductance of an interacting sample to its intrinsic properties, without taking into account the way it is connected to the electrodes.

While clean interacting systems are of physical interest (e.g., carbon nanotubes), it is also important to consider the generic case of disordered systems. To this end, we add the term $W \sum_{i=1}{ }^{L_{\mathrm{S}}}{ }_{v_{i}} n_{i}$ to Hamiltonian (6), where $W$ is the disorder strength and the $v_{i}$ are distributed equally in $[-1 / 2,1 / 2]$. We have checked that $D$ scales with $L$ as before [Eq. (7)], ensuring a well-defined limiting value for $g$. The even-odd dependence of $g$ disappears when disorder is introduced. The combined effect of disorder and interactions on $g$ is shown in Fig. 4. In the ballistic regime (at $W=1$ the mean free path exceeds $L_{\mathrm{S}}$ ), the effect of $W$ is weak at small $U$, 


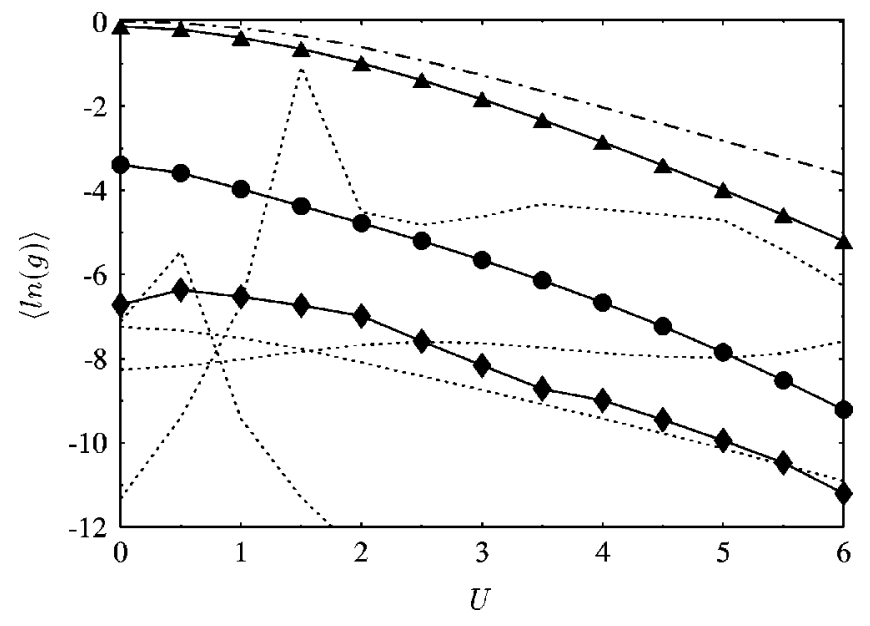

FIG. 4. Ensemble averages of $\ln g$ as a function of the interaction at $L_{\mathrm{S}}=8$, in the presence of disorders $W=1(\boldsymbol{\Delta}), 5(\mathbf{O})$, and $9(\diamond)$. The statistical errors are smaller than the symbol size. The dash-dotted line represents the clean case, the dotted lines represent four individual samples at $W=9$.

and it becomes more pronounced at stronger $U$ (when the disorder pins the Mott insulator, reinforcing the localization). At large $W, g$ for individual samples exhibits peaks as a function of $U$ whenever a charge reorganization occurs, in analogy to the persistent current calculated without the lead. ${ }^{20}$ Very remarkably, the ensemble average of $\ln g$ is increased by a moderate repulsive $U$, showing the nontrivial interplay of disorder and interactions in a transport problem.

In conclusion, starting from a recent proposal, ${ }^{10}$ we have provided a well-defined procedure for calculating the conductance $g$ of interacting one-dimensional wires, and used it to investigate correlation and disorder effects. While the interaction reduces $g$ for spinless fermions in the presence of weak or moderate disorder, a moderate repulsive interaction increases $g$ at strong disorder. We also determined the crucial role of the sample-to-lead contacts on the conductance.

After submission of our manuscript, a preprint by $\mathrm{V}$. Meden and U. Schollwöck [published as Phys. Rev. B 67, 193303 (2003)] appeared which uses an approach similar to ours. Their comparison with a numerical many-body Greenfunction technique for the conductance at not too strong interaction supports the validity of our results.

We thank O. Sushkov and C. Stafford for useful discussions, P. Schmitteckert and Ph. Brune for their DMRG programs, and V. Meden and U. Schollwöck for helpful comments. R.A.M. acknowledges the financial support from the European Union's Human Potential Program (Contract No. HPRN-CT-2000-00144). R.A.J. and D.W. thank the INT at the University of Washington for its hospitality and support during completion of this work.
${ }^{1}$ R. Landauer, IBM J. Res. Dev. 1, 223 (1957).

${ }^{2}$ M. Büttiker, Phys. Rev. Lett. 57, 1761 (1986).

${ }^{3}$ Y. Imry, Introduction to Mesoscopic Physics (Oxford University Press, New York, 1997).

${ }^{4}$ P. Prelovsek and X. Zotos, cond-mat/0203303 (unpublished).

${ }^{5}$ Y. Meir and N.S. Wingreen, Phys. Rev. Lett. 68, 2512 (1992).

${ }^{6}$ H.M. Pastawski, Phys. Rev. B 44, 6329 (1991).

${ }^{7}$ R. Berkovits and Y. Avishai, Phys. Rev. Lett. 76, 291 (1996).

${ }^{8}$ P.L. McEuen, M.S. Fuhrer, and H. Park, IEEE Trans. Nanotech. 1, 78 (2002), and references therein.

${ }^{9}$ See, e.g., Chem. Phys. 281, 111-487 (2002), special issue on Transport in Molecular Wires, edited by P. Hänggi, M. Ratner, and S. Yaliraki.

${ }^{10}$ O.P. Sushkov, Phys. Rev. B 64, 155319 (2001).

${ }^{11}$ I. Safi and H.J. Schulz, Phys. Rev. B 52, R17 040 (1995).

${ }^{12}$ L. P. Kouwenhoven, C. M. Marcus, P. L. McEuen, S. Tarucha, R. M. Westervelt, and N. S. Wingreen, in Mesoscopic Electron Transport, edited by L. L. Sohn, L. P. Kouwenhoven, and G. Schön (Kluwer Academic, Dordrecht, 1997).

${ }^{13}$ W. Kohn, Phys. Rev. 133, A171 (1964).
${ }^{14}$ D.J. Thouless, Phys. Rev. Lett. 39, 1167 (1977).

${ }^{15}$ U. Eckern and P. Schwab, Adv. Phys. 44, 387 (1995).

${ }^{16}$ B.S. Shastry and B. Sutherland, Phys. Rev. Lett. 65, 243 (1990).

${ }^{17}$ R.M. Fye, M.J. Martins, D.J. Scalapino, J. Wagner, and W. Hanke, Phys. Rev. B 44, 6909 (1991).

${ }^{18}$ S.R. White, Phys. Rev. B 48, 10345 (1993).

${ }^{19}$ P. Schmitteckert, Ph.D. thesis, Universität Augsburg, 1996.

${ }^{20}$ P. Schmitteckert, R.A. Jalabert, D. Weinmann, and J.-L. Pichard, Phys. Rev. Lett. 81, 2308 (1998).

${ }^{21}$ H.-F. Cheung, Y. Gefen, E.K. Riedel, and W.-H. Shih, Phys. Rev. B 37, 6050 (1988).

${ }^{22}$ For a quantum dot in the Coulomb blockade regime [C.A. Stafford, R. Kotlyar, and S. Das Sarma, Phys. Rev. B 58, 7091 (1998)], one obtains $g \propto J^{2}(\pi / 2)$ and $g \propto D^{2}$.

${ }^{23}$ A. Oguri, Phys. Rev. B 59, 12240 (1999).

${ }^{24}$ C.L. Kane and M.P.A. Fisher, Phys. Rev. Lett. 68, 1220 (1992).

${ }^{25}$ A.O. Gogolin and N.V. Prokof'ev, Phys. Rev. B 50, 4921 (1994).

${ }^{26}$ In the noninteracting derivation of Eq. (5), the first corrections in $1 / L$ and $\tau_{W}$ have opposite sign. A potential well accelerates the particles leading to $\tau_{W}<0$, and $C>0$. 